\title{
Eficácia de herbicidas aplicados em plantas adultas de Crotalaria spectabilis e Crotalaria ochroleuca ${ }^{1}$
}

\section{Herbicides efficacy applied in Crotalaria spectabilis and Crotalaria ochroleuca adult plants}

\author{
Miriam Hiroko Inoue ${ }^{2}$; Júlio César Barbosa Duarte ${ }^{3}$; Kassio Ferreira Mendes ${ }^{4}$; Juliano Sztoltz ${ }^{3}$; \\ Ronei Ben ${ }^{3}$; Rafael Luiz Pereira ${ }^{3}$
}

Resumo - Objetivou-se com este trabalho avaliar eficácia no controle de herbicidas aplicados em plantas adultas de Crotalaria spectabilis e Crotalaria ochroleuca. Foram instalados três experimentos com sete herbicidas dispostos em delineamento de blocos ao acaso com quatro repetições. Foram realizadas avaliações visuais de controle aos 3, 7, 14, 21, 35 e 42 dias após a aplicação (DAA) e de rebrota aos 21, 28, 35 e 42 (DAA). Concluiu-se que o tratamento com aplicação sequencial de glyphosate $\left(1.550 / 1.860 \mathrm{~g} \mathrm{ha}^{-1}\right)$ proporcionou controle satisfatório para ambas espécies de Crotalaria. Os tratamentos glyphosate + 2,4-D $\left(1.240+806 \mathrm{~g} \mathrm{ha}^{-1}\right)$, glyphosate + flumioxazina $\left(1.240+50 \mathrm{~g} \mathrm{ha}^{-1}\right)$ e glyphosate + metsulfuron-methyl $(1.240+1,8 \mathrm{~g}$ $\mathrm{ha}^{-1}$ ) foram eficientes somente para o controle de C. spectabilis, enquanto que os glyphosate + carfentrazone-ethyl $\left(1.240+32 \mathrm{~g} \mathrm{ha}^{-1}\right)$ e glyphosate + saflufenacil $\left(1.240+52,5 \mathrm{~g} \mathrm{ha}^{-1}\right)$ foram eficazes para o controle de $C$. ochroleuca. O tratamento glyphosate + chlorimuron-methyl (1.240 $+10 \mathrm{~g} \mathrm{ha}^{-1}$ ) não foi eficiente para nenhuma das espécies de crotalária. Independente das doses de flumioxazina $\left(20 ; 30 ; 40 ; 50\right.$; e $\left.60 \mathrm{~g} \mathrm{ha}^{-1}\right)$ não foi constatada eficácia de controle para $C$. spectabilis.

Palavras-chaves: Dessecação, rotação de culturas, glyphosate, flumioxazina

\begin{abstract}
The objective of this study was to evaluate the efficacy in herbicides control applied in Crotalaria spectabilis and Crotalaria ochroleuca adult plants. Three experiments were installed with seven herbicides arranged in a randomized block design with four repetitions. It was realized visual evaluations of control at 3,7,14,21, 35 and 42 after applications (DAA), and regrowth at 21, 28, 35 and 42 (DAA). It was concluded that treatment with sequential application of glyphosate $\left(1,550+1,860 \mathrm{~g} \mathrm{ha}^{-1}\right)$ provided satisfactory control for both Crotalaria species. The treatments glyphosate $+2,4-\mathrm{D}\left(1,240+806 \mathrm{~g} \mathrm{ha}^{-1}\right)$, glyphosate + flumioxazina $(1,240+50 \mathrm{~g}$ $\left.\mathrm{ha}^{-1}\right)$ and glyphosate + metsulfuron-methyl $\left(1,240+1.8 \mathrm{~g} \mathrm{ha}^{-1}\right)$, were efficient only for controlling C. spectabilis, while glyphosate + carfentrazone-ethyl $\left(1,240+32 \mathrm{~g} \mathrm{ha}^{-1}\right)$ and glyphosate + saflufenacil $\left(1,240+52.5 \mathrm{~g} \mathrm{ha}^{-1}\right)$ were efficient for controlling C. ochroleuca.

\footnotetext{
${ }^{1}$ Recebido para publicação em 27/01/2012 e aceito em 11/08/2012.

${ }^{2}$ Docente do Departamento de Agronomia da Universidade do Estado de Mato Grosso (UNEMAT), Rodovia MT 358, Km 7, CEP: 78300-000, Tangará da Serra, MT. E-mail: <miriamhinoue@ hotmail.com>;

${ }^{3}$ Discentes do curso de Agronomia da Universidade do Estado de Mato Grosso (UNEMAT), Tangará da Serra, MT;

${ }^{4}$ Mestrando do Programa de Pós-Graduação em Agronomia (Produção Vegetal) da Universidade Federal de Viçosa (UFV), Rio Paranaíba, MG.
} 
Glyphosate + chlorimuron-methyl $\left(1,240+10 \mathrm{~g} \mathrm{ha}^{-1}\right)$ treatment was not efficient for any species of sun hemp. Independent of flumioxazina doses $\left(20 ; 30 ; 40 ; 50\right.$; and $\left.60 \mathrm{~g} \mathrm{ha}^{-1}\right)$ it was not verified the effectiveness of control for $C$. spectabilis.

Keywords: Burndown, crop rotation, glyphosate, flumioxazina

\section{Introdução}

A expansão da área ocupada pela agricultura na região do cerrado tem acarretado o surgimento de áreas que, além da degradação, sofrem severas infestações por plantas daninhas e pragas de solo, sendo muitas vezes abandonadas por se tornarem improdutivas (Hayashi et al., 2002).

Uma das alternativas para o desenvolvimento agrícola sustentável do cerrado pode ser o uso da adubação verde, buscando não apenas a reposição dos elementos como o nitrogênio, o fósforo e o potássio, como também a melhoria das propriedades físicas do solo (Santos \& Campelo Jr., 2003). A crotalária é considerada planta de cobertura verde, que vem sendo bastante estudada, com potencial de proteção do solo e atuar no controle de plantas daninhas (Nascimento et al., 2005). Já Boghossian et al. (2007), destacaram a importância destas espécies na agricultura pela capacidade de fixação de nitrogênio atmosférico no solo.

No entanto, é importante ressaltar o interesse dos produtores rurais pelo seu cultivo, com o propósito de complementar os métodos tradicionais de controle de nematóides, minimizando o custo de produção. Segundo Inomoto et al. (2008), a ocorrência destra praga de solo em culturas anuais (principalmente a soja, feijão, milho e cana de açúcar) tem crescido a nível preocupante na região central do Brasil.

Crotalaria spectabilis e Crotalaria ochroleuca, da família Fabaceae, são utilizadas em sistemas de rotação de cultura, adubação verde, cobertura morta, fixação de nitrogênio atmosférico e controle de nematóides na agricultura, além de reduzirem significativamente o número e peso da matéria seca da população de plantas daninhas, tais como Digitaria horizontalis, Hyptis lophanta e Amaranthus spinosus (Erasmo et al., 2004).

Apesar da importância dos benefícios que algumas espécies de crotalária apresentam ao sistema de produção agrícola, essas ainda causam grande apreensão aos técnicos e produtores que estão trabalhando com essas plantas no Brasil. Tal fato ocorre, visto que até o momento ainda não existem recomendações concretas de herbicidas para o manejo de dessecação de plantas adultas dessas espécies. Esta afirmação é comprovada por Espanhol et al. (2011) que em observações em campo encontraram dificuldade no controle químico de plantas adultas de Crotalaria juncea em áreas de plantio direto no momento da dessecação.

Contudo, Oliveira Neto et al. (2011) em experimento de controle químico de Crotalaria spectabilis testando glyphosate + 2,4-D em mistura $(2.160+3.350 ; 2.880+2.680$ e 3.600 $+2.010 \mathrm{~g} \mathrm{ha}^{-1}$ ), verificaram que não houve diferença significativa de controle aos 28 DAA, apresentando níveis de controle de acima de 97,5\% para todas as doses desta mistura. No entanto, no mesmo experimento, os autores testaram três doses de 2,4-D isolado $\left(2.010 ; 2.680\right.$ e $\left.3.350 \mathrm{~g} \mathrm{ha}^{-1}\right)$ e verificaram níveis de controle aos 28 DAA inferiores aos anteriores, porém com diferença significativa entre as doses, que foi de $40,8 \% ; 48,8 \%$ e $71,5 \%$, respectivamente.

Arizaleta et al. (2008), observaram que a mistura de glyphosate + metsulfuron-methyl tem sido eficiente na redução da densidade de plantas daninhas. Entretanto, Espanhol et al. (2011), relataram controles superiores a $80 \%$ para plantas de Crotalaria juncea aos 28 DAA de glyphosate + metsulfuron-methyl $(1620+8$ 
$\left.\mathrm{g} \mathrm{ha}^{-1}\right)$ e glyphosate $+2,4-\mathrm{D}\left(1620+1209 \mathrm{~g} \mathrm{ha}^{-}\right.$

$\left.{ }^{1}\right)$, apesar de ter ocorrido um elevado número de rebrota das plantas dessecadas. Com base nestas informações, o trabalho teve como objetivo avaliar a eficácia de diferentes herbicidas e doses, em aplicações isoladas e em misturas na dessecação de plantas adultas de Crotalaria spectabilis e Crotalaria ochroleuca.

\section{Material e Métodos}

Foram realizados três experimentos em condições de campo, em duas áreas distintas, ambas cultivadas com Crotalaria spectabilis e Crotalaria ochroleuca em rotação com a cultura da soja. Os experimentos 1 e 3 foram realizados na Fazenda Jatobá, situada em Deciolândia, distrito do município de Diamantino-MT, apresentando como coordenadas geográficas $57^{\circ} 30^{\prime} 51^{\prime \prime}$ W e $14^{\circ} 11^{\prime} 37^{\prime \prime}$ S e à $624 \mathrm{~m}$ de altitude. $\mathrm{O}$ solo da área é classificado como Latossolo Vermelho, de textura argilosa (Embrapa, 2006). O Experimento 2 foi conduzido na Fazenda Barra Bonita, situada no município de São José do Rio Claro-MT, apresentando como coordenadas geográficas $57^{\circ} 06^{\prime} 57^{\prime \prime}$ W e $13^{\circ} 50^{\prime} 22^{\prime \prime} \mathrm{S}$ e à $403 \mathrm{~m}$ de altitude. $\mathrm{O}$ solo da área é classificado como Latossolo Vermelho Amarelo podzólico distrófico, de textura argilosa (Embrapa 2006).

Nos experimentos 1 e 2, foram avaliados sete tratamentos e uma testemunha. Os tratamentos consistiram de aplicações sequenciais e associações dos herbicidas glyphosate, 2,4-D, flumioxazina, metsulfuronmethyl, carfentrazone-ethyl, chlorimuronmethyl e saflufenacil visando a dessecação de plantas adultas de crotalária (Tabela 1 ).

Tabela 1. Herbicidas e suas respectivas doses, para dessecação de plantas adultas de Crotalaria spectabilis (Experimento 1) e Crotalaria ochroleuca (Experimento 2).

\section{Tratamentos}

Doses (g e.a. ou i.a. ha $^{-1}$ )

1. Testemunha sem herbicida

2. glyphosate/glyphosate ${ }^{1}$

$1.550 / 1.860$

3. glyphosate $+2,4-\mathrm{D}$

$1.240+806$

4. glyphosate + flumioxazina

$1.240+50$

5. glyphosate + metsulfuron-methyl

$1.240+1,8$

6. glyphosate + carfentrazone-ethyl

$1.240+32$

7. glyphosate + chlorimuron-methyl

$1.240+10$

8. glyphosate + saflufenacil

$1.240+52,5$

"Aplicação seguencial.

No Experimento 3 os tratamentos consistiram na aplicação em pós-emergência de Crotolaria spectabilis de cinco doses do herbicida flumioxazina $(20 ; 30 ; 40 ; 50 ;$ e $60 \mathrm{~g}$ $\mathrm{ha}^{-1}$ ) com adição na calda de $0,5 \%$ do óleo mineral Iharol ${ }^{\circledR}$, além de uma testemunha sem herbicida, totalizando seis tratamentos.

Os três experimentos foram dispostos em delineamento de blocos ao acaso com quatro repetições, em que as unidades experimentais apresentaram área total de $18 \mathrm{~m}^{2}$ (3 x $6 \mathrm{~m}$ ), excluindo-se $0,5 \mathrm{~m}$ das laterais e das extremidades das parcelas nas avaliações, o que conferiu uma área útil de $10 \mathrm{~m}^{2}$.

Os experimentos foram realizados no período de março a agosto de 2011. Para os experimentos 1 e 3, foram semeadas sementes de Crotalaria spectabilis no dia 18 de março de 2011, em sistema de plantio direto, no espaçamento de $0,5 \mathrm{~m}$ entre linhas com gasto de aproximadamente $16 \mathrm{~kg}$ de sementes ha ${ }^{-1}$. Já para a realização do Experimento 2 foi semeada a lanço sementes de Crotalaria ochroleuca no dia 14 de março de 2011, com 
gasto de aproximadamente $12 \mathrm{~kg}$ de sementes $\mathrm{ha}^{-1}$. Em ambos os casos, a adubação seguiu o padrão da propriedade.

Nos experimentos 1 e 3 os tratamentos foram aplicados aos 93 dias após a semeadura (DAS) da crotalária, quando as plantas apresentavam em média $0,85 \mathrm{~m}$ de altura. Já no Experimento 2 os tratamentos foram aplicados aos 97 DAS da crotalária, quando as plantas apresentavam em média $1,55 \mathrm{~m}$ de altura. Nos três experimentos as plantas se encontravam em estádio de pleno florescimento na ocasião da aplicação. As condições climáticas no momento da aplicação dos tratamentos dos experimentos 1 e 2 foram de $28,5{ }^{\circ} \mathrm{C}$ de temperatura do ar, velocidade dos ventos de $3,17 \mathrm{~km} \mathrm{~h}^{-1}$ e umidade relativa do ar de aproximadamente 35\%. Para o Experimento 3, a temperatura e umidade relativa do ar foram, em média, praticamente semelhantes as observadas nos experimentos 1 e 2, diferenciando apenas a velocidade dos ventos que foi de $4,68 \mathrm{~km} \mathrm{~h}^{-1}$.

Para as aplicações dos tratamentos foi utilizado um pulverizador costal pressurizado com $\mathrm{CO}_{2}$ a $28 \mathrm{lbf} \mathrm{pol}^{-2}$, munido de barra de 3 $\mathrm{m}$ composta de seis pontas de jato plano ("leque") 110.02, espaçados de 0,5 m, com consumo de calda equivalente a $150 \mathrm{~L} \mathrm{ha}^{-1}$. Em todos os tratamentos foi realizada apenas uma aplicação, com exceção ao tratamento dois nos experimentos 1 e 2, que recebeu uma aplicação sequencial sete dias após a primeira.

Após a aplicação foram realizadas avaliações visuais de controle aos $3,7,14,21$, 28, 35 e 42 dias após a aplicação (DAA), onde foram atribuídas notas em porcentagem, sendo 0 (zero) correspondente a ausência de controle e a nota $100 \%$ corresponde a morte total das plantas de acordo com a escala da SBCPD (1995). Aos 28, 35 e 42 DAA foram realizadas avaliações de rebrota dos tratamentos, utilizando-se a escala percentual de 0 a $100 \%$, significando ausência de rebrota e rebrota total das plantas, respectivamente (Procópio et al., 2006).
Os dados coletados nos experimentos 1 e 2, foram submetidos à análise de variância pelo teste $\mathrm{F}$ e suas médias significativas comparadas pelo critério de Scott-Knott a 5\% de probabilidade. No Experimento 3, os dados coletados foram submetidos a análise de variância pelo teste $\mathrm{F}$ e os efeitos das doses de flumioxazina significativas, foram submetidos à análise de regressão, escolhendo-se o modelo de melhor ajuste em função do maior coeficiente de determinação.

\section{Resultados e Discussão}

\section{Experimento 1}

No Experimento 1 (Tabela 2), a análise de variância apresentou efeito significativo pelo teste $\mathrm{F}$ a $5 \%$ de probabilidade entre os tratamentos para as sete épocas de avaliações em dias após a aplicação (DAA).

No controle percentual da Crotalaria spectabilis (Tabela 2), observa-se que, aos 3 DAA, os melhores resultados de controle foram obtidos nos tratamentos $\mathrm{T}_{6}$ e $\mathrm{T}_{8}$, com $41,25 \%$ e $39,50 \%$, respectivamente. Nos tratamentos, $\mathrm{T}_{3}, \mathrm{~T}_{4}$ e $\mathrm{T}_{5}$, não houve diferença significativa entre si. $\mathrm{O}$ mesmo ocorreu para os tratamentos $T_{2}$ e $T_{7}$, que também não diferenciaram significativamente entre si. No entanto, todos os tratamentos proporcionaram baixos níveis de controle inicial, de acordo com a classificação da SBCPD (1995).

$\mathrm{Na}$ segunda avaliação de controle realizada aos 7 DAA (Tabela 2), todos os tratamentos diferiram entre si, com destaque novamente ao tratamento $\mathrm{T}_{8}$, que proporcionou índice de controle superior aos demais (próximo a 85\%), evidenciando o sinergismo entre o saflufenacil e glyphosate. Os dados corroboram com Belani et al. (2010), que testando saflufenacil + glyphosate, nas doses de $35,5+1.080 \mathrm{~g} \mathrm{ha}^{-1}$ para o controle de plantas adultas de Conyza bonariensis, encontrou resultados de $88 \%$ aos 7 DAA. O segundo melhor nível de controle foi apresentado pelo tratamento $\mathrm{T}_{6}$, com 
aproximadamente $80 \%$. Os demais tratamentos

abaixo de $60 \%$.

controlaram a Crotalaria spectabilis em nível

Tabela 2. Porcentagem de controle de plantas adultas de Crotalaria spectabilis em dias após a aplicação (DAA) dos tratamentos. Deciolândia - MT, 2011.

\begin{tabular}{|c|c|c|c|c|c|c|c|c|c|c|c|c|c|c|}
\hline TRAT & \multicolumn{14}{|c|}{ Dias após a aplicação (DAA) } \\
\hline & 3 & & 7 & & \multicolumn{2}{|c|}{14} & \multicolumn{2}{|c|}{21} & \multicolumn{2}{|l|}{28} & \multicolumn{2}{|l|}{35} & \multicolumn{2}{|l|}{42} \\
\hline 1 & 0,00 & $\bar{d}$ & 0,00 & $\mathrm{~g}$ & 0,00 & $\bar{f}$ & 0,00 & f & 0,00 & $\bar{d}$ & 0,00 & $\overline{\mathrm{e}}$ & 0,00 & $\overline{\mathrm{d}}$ \\
\hline 2 & 31,75 & $\mathrm{c}$ & 54,75 & $\mathrm{~d}$ & 72,75 & $\mathrm{c}$ & 98,25 & $\mathrm{a}$ & 97,75 & $\mathrm{a}$ & 98,25 & $\mathrm{a}$ & 97,75 & $\mathrm{a}$ \\
\hline 3 & 36,75 & $\mathrm{~b}$ & 46,00 & $\mathrm{e}$ & 75,75 & $\mathrm{c}$ & 97,75 & $\mathrm{a}$ & 97,00 & $\mathrm{a}$ & 97,50 & $\mathrm{a}$ & 97,50 & $\mathrm{a}$ \\
\hline 4 & 35,25 & $b$ & 54,75 & $\mathrm{~d}$ & 71,50 & $\mathrm{c}$ & 93,50 & $\mathrm{~b}$ & 91,25 & $\mathrm{a}$ & 89,75 & b & 89,00 & $b$ \\
\hline 5 & 35,75 & $\mathrm{~b}$ & 59,00 & $\mathrm{c}$ & 63,50 & $\mathrm{~d}$ & 94,75 & $\mathrm{~b}$ & 92,00 & $\mathrm{a}$ & 92,25 & $\mathrm{~b}$ & 90,75 & $\mathrm{~b}$ \\
\hline 6 & 41,25 & $\mathrm{a}$ & 81,00 & $\mathrm{~b}$ & 87,50 & $\mathrm{~b}$ & 76,50 & $\mathrm{c}$ & 59,00 & $\mathrm{~b}$ & 57,75 & $\mathrm{c}$ & 51,00 & $\mathrm{c}$ \\
\hline 7 & 32,00 & $\mathrm{c}$ & 34,25 & $\mathrm{f}$ & 55,50 & e & 63,25 & $\mathrm{e}$ & 50,00 & $\mathrm{c}$ & 48,75 & $\mathrm{~d}$ & 44,50 & $\mathrm{c}$ \\
\hline 8 & 39,50 & $\mathrm{a}$ & 84,75 & $\mathrm{a}$ & 95,00 & $\mathrm{a}$ & 72,25 & $\mathrm{~d}$ & 59,25 & $\mathrm{~b}$ & 57,25 & $\mathrm{c}$ & 50,50 & c \\
\hline $\mathrm{CV} \%$ & 6,63 & & 1,86 & & 6,24 & & 3,17 & & 6,77 & & 5,83 & & 7,77 & \\
\hline
\end{tabular}

- Médias seguidas de mesma letra na coluna não diferem entre si pelo teste de Scott-Knott, a 5\% de probabilidade.

$* \mathrm{~T}_{1}=$ testemunha, $\mathrm{T}_{2}=$ glyphosate $\left(1.550 / 1.860 \mathrm{~g} \mathrm{ha}^{-1}\right), \mathrm{T}_{3}=$ glyphosate $+2,4-\mathrm{D}\left(1.240+806 \mathrm{~g} \mathrm{ha}^{-1}\right), \mathrm{T}_{4}=$ glyphosate + flumioxazina $\left(1.240+50 \mathrm{~g} \mathrm{ha}^{-1}\right), \mathrm{T}_{5}=$ glyphosate + metsulfuron-methyl $\left(1.240+1,8 \mathrm{~g} \mathrm{ha}^{-1}\right), \mathrm{T}_{6}=$ glyphosate + carfentrazone-ethyl $\left(1.240+32 \mathrm{~g} \mathrm{ha}^{-1}\right), \mathrm{T}_{7}=$ glyphosate + chlorimuron-methyl $\left(1.240+10 \mathrm{~g} \mathrm{ha}^{-1}\right) \mathrm{e} \mathrm{T}_{8}$ $=$ glyphosate + saflufenacil $\left(1.240+52,5 \mathrm{~g} \mathrm{ha}^{-1}\right)$.

Aos 14 DAA (Tabela 2) o tratamento $\mathrm{T}_{8}$ ainda se destacou dos demais chegando ao nível de $95 \%$ de controle. Novamente estes resultados corroboraram com os mesmos encontrados por Belani et al. (2010) aos 14 DAA. Dessa forma, é possível verificar a semelhança no efeito da associação entre os herbicidas glyphosate e saflufenacil. $\mathrm{O}$ tratamento $\mathrm{T}_{6}$ proporcionou o segundo melhor nível de controle nesta data $(87,5 \%)$. Tal nível de controle também foi verificado por Fornarolli et al. (2010), em trabalhos realizados para o controle de Ipomoea triloba, com a associação de glyphosate + carfentrazoneethyl, $\left(1.080+20 \mathrm{~g} \mathrm{ha}^{-1}\right)$ aos 12 DAA. Nesta data de avaliação houve uma semelhança entre os tratamentos $T_{2}, T_{3}$ e $T_{4}$, que não diferiram significativamente entre si.

No entanto, é importante ressaltar que o $\mathrm{T}_{2}$ recebeu uma segunda aplicação sequencial aos 7 dias após a primeira aplicação. Quanto aos demais tratamentos obtiveram níveis de controle abaixo do aceitável (55,5\% a 63,5\%).
Aos 21 DAA (Tabela 2) os tratamentos $\mathrm{T}_{2}$ e $\mathrm{T}_{3}$ obtiveram grande desempenho, atingindo controle superior a 97\%. Esta eficiência de controle em ambos os tratamentos se mantive constantes até os 42 DAA. Oliveira Neto et al. (2011), testando diferentes doses da mistura de glyphosate $+2,4-\mathrm{D}$, para o controle de Crotalaria spectabilis encontraram resultados semelhantes aos 21 e 28 DAA, nas doses de $2.160+3.350 \mathrm{~g} \mathrm{ha}^{-1}$. O desempenho positivo também foi verificado para os tratamentos $\mathrm{T}_{4}$ e $\mathrm{T}_{5}$, que atingiram níveis de controle próximos a 94\%. Quanto ao tratamento $\mathrm{T}_{6}$, o mesmo ficou abaixo de $80 \%$, e continuou em declínio chegando a $51 \%$ aos 42 DAA.

Os resultados de rebrota das plantas de Crotalaria spectabilis sob efeito residual dos herbicidas podem ser observados na Tabela 3. Verifica-se que os níveis de rebrota foram inversamente proporcionais aos níveis de controle encontrados na Tabela 2, nas quatro avaliações realizadas. 
Tabela 3. Porcentagem de rebrota de Crotalaria spectabilis em dias após a aplicação (DAA) dos tratamentos. Deciolândia - MT, 2011.

\begin{tabular}{|c|c|c|c|c|c|c|c|c|}
\hline \multirow{3}{*}{$\begin{array}{c}\text { TRAT } \\
1\end{array}$} & \multicolumn{8}{|c|}{ Dias após a aplicação (DAA) } \\
\hline & \multicolumn{2}{|c|}{21} & \multicolumn{2}{|c|}{28} & \multicolumn{2}{|c|}{35} & \multicolumn{2}{|c|}{42} \\
\hline & 100,00 & $\mathrm{a}$ & 100,00 & $\mathrm{a}$ & 100,00 & $\mathrm{a}$ & 100,00 & $\mathrm{~A}$ \\
\hline 2 & 1,50 & $\mathrm{f}$ & 1,75 & d & 2,00 & $\mathrm{~d}$ & 2,00 & $\mathrm{E}$ \\
\hline 3 & 2,00 & $\mathrm{f}$ & 2,25 & d & 2,50 & $\mathrm{~d}$ & 2,50 & $\mathrm{e}$ \\
\hline 4 & 5,00 & $\mathrm{e}$ & 8,25 & d & 10,25 & $\mathrm{c}$ & 11,00 & d \\
\hline 5 & 4,75 & $\mathrm{e}$ & 8,50 & d & 7,75 & $\mathrm{c}$ & 9,25 & d \\
\hline 6 & 23,00 & d & 43,75 & $\mathrm{~b}$ & 42,00 & $\mathrm{~b}$ & 47,50 & $\mathrm{c}$ \\
\hline 7 & 37,75 & b & 45,25 & b & 46,25 & $\mathrm{~b}$ & 61,50 & b \\
\hline 8 & 27,75 & $\mathrm{c}$ & 40,00 & $\mathrm{c}$ & 42,25 & $\mathrm{~b}$ & 47,50 & $\mathrm{c}$ \\
\hline $\mathrm{CV} \%$ & 7,4 & & 16 , & & 9, & & 14,5 & \\
\hline
\end{tabular}

- Médias seguidas de mesma letra na coluna não diferem entre si pelo teste de Scott-Knott, a 5\% de probabilidade.

$* \mathrm{~T}_{1}=$ testemunha, $\mathrm{T}_{2}=$ glyphosate $\left(1.550 / 1.860 \mathrm{~g} \mathrm{ha}^{-1}\right), \mathrm{T}_{3}=$ glyphosate $+2,4-\mathrm{D}\left(1.240+806 \mathrm{~g} \mathrm{ha}^{-1}\right), \mathrm{T}_{4}=$ glyphosate + flumioxazina $\left(1.240+50 \mathrm{~g} \mathrm{ha}^{-1}\right), \mathrm{T}_{5}=$ glyphosate + metsulfuron-methyl $\left(1.240+1,8 \mathrm{~g} \mathrm{ha}^{-1}\right), \mathrm{T}_{6}=$ glyphosate + carfentrazone-ethyl $\left(1.240+32 \mathrm{~g} \mathrm{ha}^{-1}\right), \mathrm{T}_{7}=$ glyphosate + chlorimuron-methyl $\left(1.240+10 \mathrm{~g} \mathrm{ha}^{-1}\right) \mathrm{e} \mathrm{T}_{8}$ $=$ glyphosate + saflufenacil $\left(1.240+52,5 \mathrm{~g} \mathrm{ha}^{-1}\right)$.

Nos tratamentos $T_{2}$ e $T_{3}$, não houve diferença significativa entre eles, independente da época avaliada, mantendo níveis de rebrota entre $1,5 \%$ a $2,5 \%$ durante os quatro períodos. Embora os tratamentos $\mathrm{T}_{4}$ e $\mathrm{T}_{5}$, apresentem a mesma tendência dos tratamentos anteriores, os níveis de rebrota foram maiores, variando entre $4,75 \%$ e $11,00 \%$. Os tratamentos que obtiveram resultados insatisfatórios foram $\mathrm{T}_{6}$, $\mathrm{T}_{7}$, e $\mathrm{T}_{8}$, pois a partir dos 21 DAA, houve ascensão nos níveis de rebrota, chegando aos 42 DAA com valores acima de 47\%. Tais resultados de 47,50\%, 61,50\% e 47,50\%, respectivamente, sugerem que plantas de Crotalaria spectabilis são menos susceptíveis ao herbicida glyphosate em mistura com carfentrazone-ethyl, chlorimuron-methyl e saflufenacil, nas condições que foram realizados os experimentos.

Espanhol et al. (2011) avaliando da a ação do glyphosate em aplicação isolada e em misturas com 2,4-D ou metsulfuron-methyl na dessecação de plantas adultas de Crotalaria juncea relataram resultados de rebrota superiores aos deste trabalho. Os autores verificaram níveis de rebrota acima de $70 \%$ aos 42 DAA, nos tratamentos que tiveram uma aplicação isolada de glyphosate, na mistura de glyphosate $+2,4-\mathrm{D}$ e de glyphosate + metsulfuron-methyl, nas doses de $1,62 \mathrm{~kg} \mathrm{ha}^{-1}$; $1,62+1,21 \mathrm{~kg} \mathrm{ha}^{-1}$ e $1,62+8 \mathrm{~g} \mathrm{ha}^{-1}$, respectivamente.

\section{Experimento 2}

No experimento 2 (Tabela 4), a análise de variância apresentou efeito significativo pelo teste $\mathrm{F}$ a $5 \%$ de probabilidade entre os tratamentos.

Aos 14 DAA (Tabela 4), observou-se semelhança nos níveis de controle entre os tratamentos $\mathrm{T}_{2}, \mathrm{~T}_{3}, \mathrm{~T}_{4}, \mathrm{~T}_{6}$, e $\mathrm{T}_{8}$, cujos valores foram entre $85,75 \%$ e $89,75 \%$. Os tratamentos $\mathrm{T}_{5}$ e $\mathrm{T}_{7}$ obtiveram níveis acima de $70 \%$. Quanto a avaliação realizada aos 21 DAA, observou-se que não houve diferença significativa entre os tratamentos, exceto para o tratamento $\mathrm{T}_{2}$ que obteve o nível de controle superior aos demais com $99 \%$.

Contudo, apesar do restante dos tratamentos proporcionarem níveis de controle inferiores ao tratamento $\mathrm{T}_{2}$, todos tiveram um excelente controle com níveis acima de $95 \%$. Resultados semelhantes foram encontrados por Monquero et al. (2001) aos 14 DAA, visando determinar o tipo de interação do herbicida glyphosate em mistura com chlorimuron- 
methyl, flumioxazina e carfentrazone-ethyl no controle da planta daninha Ipomoea grandifolia.

Tanto no Experimento 1 quanto no Experimento 2 (Tabelas 3 e 4), observou-se que o tratamento $T_{2}$ (aplicação sequencial de glyphosate), por sua vez, apresentou ação inicial mais lenta, igualando-se aos demais tratamentos aos 14 DAA. Este resultado condiz com Rodrigues \& Almeida (2011), que afirmam que os sintomas resultantes da aplicação do glyphosate são lentos, iniciando pelo amarelecimento progressivo das folhas, murchamento com posterior necrose e morte das plantas que pode demorar de 4 a 20 dias, conforme a espécie e estádio fenológico.

Tabela 4. Porcentagem de controle de plantas adultas de Crotalaria ochroleuca em dias após a aplicação (DAA) dos tratamentos. São José do Rio Claro - MT, 2011.

\begin{tabular}{|c|c|c|c|c|c|c|c|c|c|c|c|c|c|c|}
\hline TRAT & \multicolumn{14}{|c|}{ Dias após a aplicação (DAA) } \\
\hline & 3 & & 7 & & 14 & & 21 & & 28 & & 35 & & 42 & \\
\hline 1 & 0,00 & $\mathrm{f}$ & 0,00 & $\mathrm{e}$ & 0,00 & $\mathrm{~d}$ & 0,00 & $\mathrm{c}$ & 0,00 & $\mathrm{~g}$ & 0,00 & $\mathrm{e}$ & 0,00 & $\mathrm{e}$ \\
\hline 2 & 20,75 & $\mathrm{e}$ & 51,00 & $\mathrm{c}$ & 89,25 & $\mathrm{a}$ & 99,00 & $\mathrm{a}$ & 98,50 & $\mathrm{a}$ & 99,50 & $\mathrm{a}$ & 99,50 & $\mathrm{a}$ \\
\hline 3 & 34,75 & $\mathrm{a}$ & 63,75 & $\mathrm{a}$ & 85,75 & $\mathrm{a}$ & 96,00 & $b$ & 75,75 & $f$ & 75,00 & $d$ & 74,25 & $\mathrm{~d}$ \\
\hline 4 & 25,50 & $\mathrm{c}$ & 57,50 & $b$ & 88,75 & $\mathrm{a}$ & 96,75 & b & 83,50 & $\mathrm{~d}$ & 80,25 & $\mathrm{c}$ & 78,75 & $\mathrm{c}$ \\
\hline 5 & 22,50 & d & 51,25 & $\mathrm{c}$ & 79,25 & b & 96,50 & b & 76,25 & $\mathrm{f}$ & 75,25 & d & 75,25 & d \\
\hline 6 & 26,50 & b & 52,00 & $c$ & 89,00 & $\mathrm{a}$ & 97,25 & b & 90,50 & $\mathrm{c}$ & 90,00 & b & 90,00 & $\mathrm{~b}$ \\
\hline 7 & 24,75 & $\mathrm{c}$ & 44,75 & d & 74,00 & c & 95,50 & b & 81,25 & e & 80,75 & $\mathrm{c}$ & 74,75 & $\mathrm{~d}$ \\
\hline 8 & 26,75 & $\mathrm{~b}$ & 66,25 & $\mathrm{a}$ & 89,75 & $\mathrm{a}$ & 96,50 & b & 96,50 & $\mathrm{~b}$ & 89,00 & $\mathrm{~b}$ & 91,50 & $\mathrm{~b}$ \\
\hline $\mathrm{CV} \%$ & 3,55 & & 3,88 & & 2,99 & & 0,93 & & 1,60 & & 2,19 & & 1,87 & \\
\hline
\end{tabular}

- Médias seguidas de mesma letra na coluna não diferem entre si pelo teste de Scott-Knott, a 5\% de probabilidade. $* \mathrm{~T}_{1}=$ testemunha, $\mathrm{T}_{2}=$ glyphosate $\left(1.550 / 1.860 \mathrm{~g} \mathrm{ha}^{-1}\right), \mathrm{T}_{3}=$ glyphosate $+2,4-\mathrm{D}\left(1.240+806 \mathrm{~g} \mathrm{ha}^{-1}\right), \mathrm{T}_{4}=$ glyphosate + flumioxazina $\left(1.240+50 \mathrm{~g} \mathrm{ha}^{-1}\right), \mathrm{T}_{5}=$ glyphosate + metsulfuron-methyl $\left(1.240+1,8 \mathrm{~g} \mathrm{ha}^{-1}\right), \mathrm{T}_{6}=$ glyphosate + carfentrazone-ethyl $\left(1.240+32 \mathrm{~g} \mathrm{ha}^{-1}\right), \mathrm{T}_{7}=$ glyphosate + chlorimuron-methyl $\left(1.240+10 \mathrm{~g} \mathrm{ha}^{-1}\right) \mathrm{e} \mathrm{T}_{8}$ $=$ glyphosate + saflufenacil $\left(1.240+52,5 \mathrm{~g} \mathrm{ha}^{-1}\right)$.

$\mathrm{O}$ tratamento $\mathrm{T}_{2}$ manteve uma semelhança no controle nas avaliações 21 e 35 DAA, chegando aos 42 DAA com 99,5\%, ocorrendo o mesmo para os tratamentos $\mathrm{T}_{6} \mathrm{e}$ $\mathrm{T}_{8}$. Quanto ao restante dos tratamentos, houve uma redução gradativa do controle após os 21 DAA, chegando aos 42 DAA com níveis de abaixo de 80\%. Carvalho et al. (2003), avaliando a eficácia dos herbicidas glyphosate + chlorimuron-methyl $\left(960+10 \mathrm{~g} \mathrm{ha}^{-1}\right) \mathrm{e}$ glyphosate $+2,4-\mathrm{D}\left(960+868 \mathrm{~g} \mathrm{ha}^{-1}\right)$ no controle de Euphorbia heterophylla e Bidens pilosa, encontraram resultados inversos aos dos últimos tratamentos citados, pois embora tenham sido eficientes no controle das plantas daninhas, proporcionando eficiência média superiores a 91\%, os níveis máximos de controle apenas foram observados aos 45 DAA.
Na Tabela 5, estão apresentados os dados referentes à porcentagem de rebrota de plantas de adultas de Crotalaria ochroleuca após a aplicação dos tratamentos. No tratamento $\mathrm{T}_{2}$, com duas aplicações de glyphosate, nota-se uma menor porcentagem de rebrota para todas as épocas avaliadas em relação a testemunha e quando comparados aos tratamentos com glyphosate em mistura com os outros herbicidas.

Quanto aos tratamentos $\mathrm{T}_{6}$ e $\mathrm{T}_{8}$ (Tabela 5), verificou-se que houve semelhança nos níveis de rebrota, diferindo significativamente entre si apenas na segunda época avaliada, mantendo-se os níveis de rebrota abaixo de $8,5 \%$ até aos 42 DAA. Os demais tratamentos promoveram os maiores índices de rebrotas. 
Tabela 5. Porcentagem de rebrota de Crotalaria ochroleuca em dias após a aplicação (DAA) dos tratamentos. São José do Rio Claro - MT, 2011.

\begin{tabular}{|c|c|c|c|c|c|c|c|c|}
\hline \multirow[t]{2}{*}{ TRAT } & \multicolumn{8}{|c|}{ Dias após a aplicação (DAA) } \\
\hline & 21 & & 28 & & 35 & & 42 & \\
\hline 1 & 100,00 & $\bar{a}$ & 100,00 & $\bar{a}$ & 100,00 & $\bar{a}$ & 100,00 & $\mathrm{a}$ \\
\hline 2 & 1,00 & d & 1,50 & $\mathrm{~g}$ & 0,50 & $\mathrm{e}$ & 0,50 & e \\
\hline 3 & 4,25 & $\mathrm{~b}$ & 24,25 & $\mathrm{~b}$ & 25,00 & $\mathrm{~b}$ & 25,50 & $\mathrm{~b}$ \\
\hline 4 & 3,25 & $\mathrm{c}$ & 16,50 & $\mathrm{~d}$ & 20,25 & $\mathrm{c}$ & 21,25 & $\mathrm{c}$ \\
\hline 5 & 3,50 & $\mathrm{c}$ & 24,00 & $\mathrm{~b}$ & 24,75 & $\mathrm{~b}$ & 24,50 & $\mathrm{~b}$ \\
\hline 6 & 2,75 & $\mathrm{c}$ & 7,25 & e & 7,25 & $\mathrm{~d}$ & 8,25 & $\mathrm{~d}$ \\
\hline 7 & 4,50 & b & 18,75 & $\mathrm{c}$ & 23,75 & $b$ & 23,75 & $b$ \\
\hline 8 & 3,50 & c & 3,50 & $\mathrm{f}$ & 7,00 & $\mathrm{~d}$ & 7,00 & $\mathrm{~d}$ \\
\hline $\mathrm{CV} \%$ & 4,96 & & 4,84 & & 5,19 & & 4,81 & \\
\hline
\end{tabular}

- Médias seguidas de mesma letra na coluna não diferem entre si pelo teste de Scott-Knott, a 5\% de probabilidade.

$* \mathrm{~T}_{1}=$ testemunha, $\mathrm{T}_{2}=$ glyphosate $\left(1.550 / 1.860 \mathrm{~g} \mathrm{ha}^{-1}\right), \mathrm{T}_{3}=$ glyphosate $+2,4-\mathrm{D}\left(1.240+806 \mathrm{~g} \mathrm{ha}^{-1}\right), \mathrm{T}_{4}=$ glyphosate + flumioxazina $\left(1.240+50 \mathrm{~g} \mathrm{ha}^{-1}\right), \mathrm{T}_{5}=$ glyphosate + metsulfuron-methyl $\left(1.240+1,8 \mathrm{~g} \mathrm{ha}^{-1}\right), \mathrm{T}_{6}=$ glyphosate + carfentrazone-ethyl $\left(1.240+32 \mathrm{~g} \mathrm{ha}^{-1}\right), \mathrm{T}_{7}=$ glyphosate + chlorimuron-methyl $\left(1.240+10 \mathrm{~g} \mathrm{ha}^{-1}\right) \mathrm{e} \mathrm{T}_{8}$ $=$ glyphosate + saflufenacil $\left(1.240+52,5 \mathrm{~g} \mathrm{ha}^{-1}\right)$.

Embora os tratamentos $\mathrm{T}_{3}, \mathrm{~T}_{4}, \mathrm{~T}_{5}$ e $\mathrm{T}_{7}$ (Tabela 5), tenham diferido significativamente entre si nas primeiras avaliações de rebrota, chegaram ao final das avaliações com índices semelhantes, variando de $21,25 \%$ a $25,5 \%$. Comparando estes tratamentos com os tratamentos $\mathrm{T}_{6}$ e $\mathrm{T}_{8}$, percebe-se que, os mesmos não afetaram a translocação ou a absorção do glyphosate nas mesmas proporções que os restantes dos tratamentos.

Dentre as espécies avaliadas, foi possível constatar que a Crotalaria spectabilis (Tabela 2) apresentou menor sensibilidade aos herbicidas estudados quando comparada com a Crotalaria ochroleuca (Tabela 4).

As diferenças de tempo para o controle das duas espécies em estudo estão em concordância com o mecanismo de ação dos herbicidas avaliados. Analisando a Tabela 1, verifica que os herbicidas estudados se enquadraram em três grupos de mecanismos de ação distintos, sendo eles: mimetizadores da auxina (2,4-D); inibidores da enzima PROTOX (flumioxazina, carfentrazone-ethyl e saflufenacil) e inibidores da síntese de aminoácidos (ALS: metsulfuron-methyl, chlorimuron-methyl e EPSPs: glyphosate).
Notadamente, flumioxazina, carfentrazone-ethyl e saflufenacil são herbicidas de contato, com expressivos danos causados à estrutura foliar, e em curto espaço de tempo, contribuem para a baixa translocação dos herbicidas às demais partes da planta (Carvalho \& López-Ovejero, 2008). No experimento 1 (Tabela 2), nota-se aos 42 DAA que os tratamentos $\mathrm{T}_{4}, \mathrm{~T}_{6}$ e $\mathrm{T}_{8}$ proporcionaram controle de $89 \%, 51 \%$ e $50 \%$ respectivamente, enquanto que no experimento 2 (Tabela 4), os mesmos tratamentos apresentaram resultados de $78 \%, 90 \%$ e $91 \%$, respectivamente.

Quanto aos outros dois grupos de mecanismo de ação: mimetizadores da auxina (2,4-D); e inibidores da síntese de aminoácidos (ALS: metsulfuron-methyl, chlorimuronmethyl) presente neste trabalho, também foram verificadas diferenças de controle entre as duas espécies de Crotalaria estudada. A mistura dos herbicidas pode ter ocasionado essas diferenças de controle, como descrito por Correia et al. (2008), onde destacaram o efeito sinergístico, em que a fitotoxicidade do ghyphosate mais flumioxazina foram mais evidentes que os observados com a aplicação isolada dos herbicidas, ou ainda, pela atividade antagônica 
dos herbicidas no trabalho de Scherder et al. (2005).

Outros fatores ainda podem ter influenciado para ocorrência dos resultados diferente entre as duas espécies sendo: a própria arquitetura das plantas, pois apesar de pertencerem ao mesmo gênero, são plantas bem distintas; a forma que foi semeada (a lanço e em linha) também pode ter aumentado ou diminuído a exposição das plantas aos herbicidas. As condições hídricas das plantas e até mesmo a textura do solo também são fatores que interferem na absorção e translocação dos herbicidas, podendo aumentar ou diminuir a eficiência de controle.
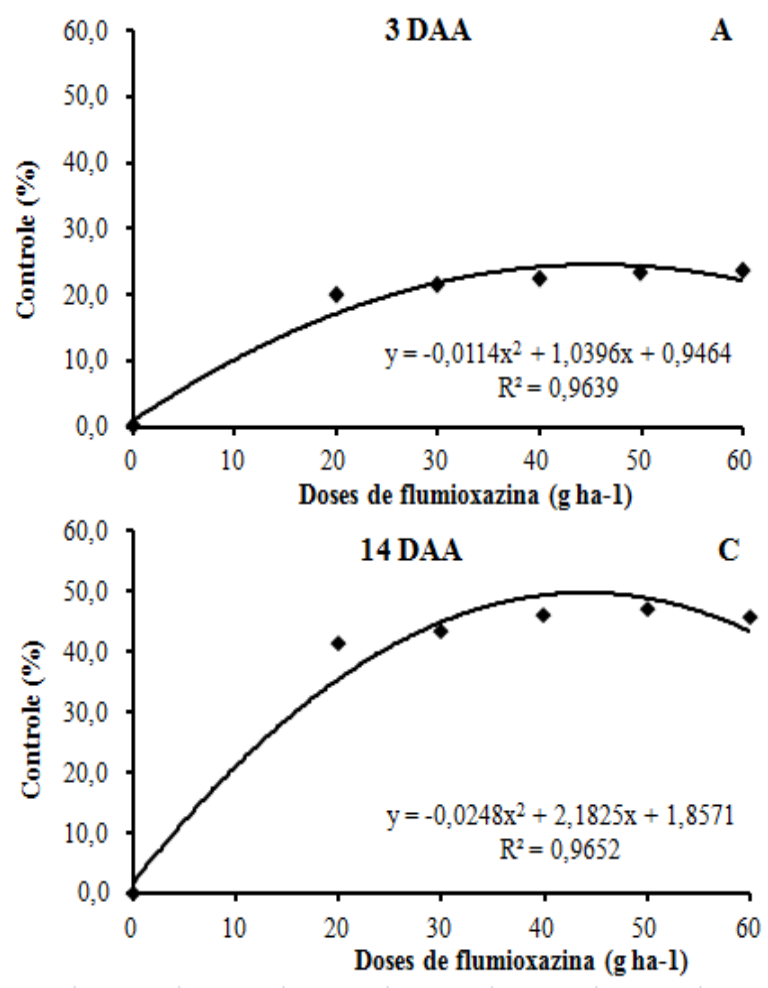

\section{Experimento 3}

Os resultados obtidos para o controle de plantas adultas de Crotalaria spectabilis no experimento 3 (Figura 1), indicaram que existem efeitos significativos das diferentes doses do herbicida flumioxazina sobre as épocas avaliadas, pelo teste $\mathrm{F}$ a $5 \%$ de probabilidade. De acordo com a Figura 1A, verifica-se que aos 3 DAA o maior controle foi proporcionado pela maior dose de flumioxazina $\left(60 \mathrm{~g} \mathrm{ha}^{-1}\right)$, porém com média não satisfatória $23,50 \%$. O efeito do controle das plantas foi quadrático e o coeficiente de determinação foi de $96,39 \%$.
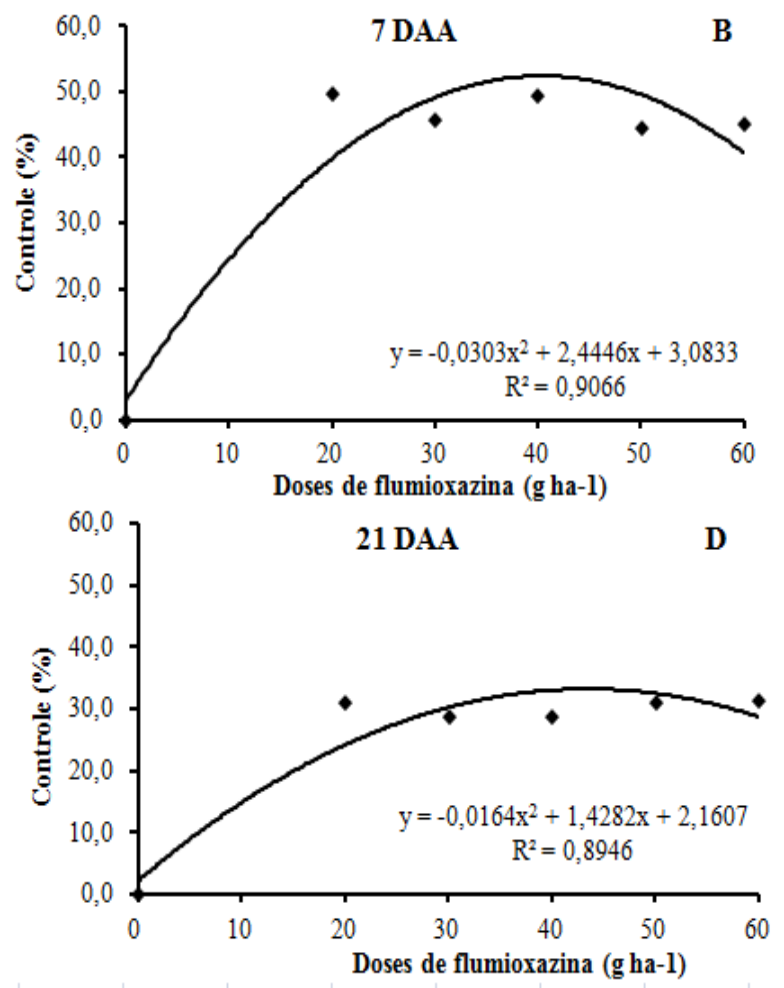

Figura 1. Porcentagem de controle visual de plantas adultas de Crotalaria spectabilis, aos 3, 7, 14 e 21 (A, B, C e D) dias após a aplicação (DAA) de seis doses em $\mathrm{g} \mathrm{ha}^{-1}$ de flumioxazina. Deciolândia - MT, 2011.

Nas duas avaliações seguintes (7 e 14 DAA), Figuras 1B e 1C, para todas as doses de flumioxazina, as porcentagens de controle foram ajustadas na forma quadrático, com linhas de tendência similares. Verifica-se que aos 7 DAA o maior controle foi proporcionado pela dose $40 \mathrm{~g} \mathrm{ha}^{-1}$, obtendo-se $49,50 \%$, enquanto que aos 14 DAA, o maior controle foi verificado pela dose $50 \mathrm{~g} \mathrm{ha}^{-1}$ com $47,25 \%$ de controle. Nas duas épocas de avaliações os 
maiores níveis de controle foram proporcionados pelas doses intermediárias. Porém com níveis bem abaixo do aceitável. Quanto à avaliação realizada aos 21 DAA, houve redução de controle para todas as doses em estudo, com destaque para a dose $60 \mathrm{~g} \mathrm{ha}^{-1}$, que proporcionou o maior controle com apenas $31,25 \%$ de eficiência.

Niekamp et al. (1999), encontraram resultados opostos ao deste trabalho visando o controle de plantas daninhas de folha larga com sulfentrazone e flumioxazina em lavoura de soja (Glycine max). Os autores verificaram que o flumioxazina $\left(90 \mathrm{~g} \mathrm{ha}^{-1}\right)$ promoveu o controle de $92 \%$ de A. theophrasti e A. artemisiiofia, $89 \%$ de X. strumarium e $96 \%$ de I. lacunosa. Resultados encontrados por Askew et al. (1999), em estudo de manejo de plantas daninhas em amendoim (Arachis hypogea) com flumioxazina em pré-emergência, verificaram que o flumioxazina (70 e $110 \mathrm{~g}$ ha ${ }^{1}$ ) controlou $96 \%$ de $S$. spinosa.

\section{Conclusões}

O herbicida glyphosate (1.550/1.860 g $\mathrm{ha}^{-1}$ ), em aplicação sequencial, foi eficiente no controle de Crotalaria spectabilis e Crotalaria ochroleuca.

As misturas em tanque de glyphosate + 2,4-D $\left(1.240+806 \mathrm{~g} \mathrm{ha}^{-1}\right)$, glyphosate + flumioxazina $\left(1.240+50 \mathrm{~g} \mathrm{ha}^{-1}\right)$ e glyphosate + metsulfuron-methyl $\left(1.240+1,8 \mathrm{~g} \mathrm{ha}^{-1}\right)$, foram eficazes somente para o controle das plantas de Crotalaria spectabilis.

Glyphosate + carfentrazone-ethyl $\left(1.240+32 \mathrm{~g} \mathrm{ha}^{-1}\right)$ e glyphosate + saflufenacil $\left(1.240+52,5 \mathrm{~g} \mathrm{ha}^{-1}\right)$ foram eficazes para o controle de Crotalaria ochroleuca.

A mistura em tanque de glyphosate + chlorimuron-methyl $\left(1.240+10 \mathrm{~g} \mathrm{ha}^{-1}\right)$ não foi eficaz para ambas as espécies de crotalária.

Independente das doses em aplicação isolada de flumioxazina, não foi constatada nenhuma eficácia de controle para as plantas adultas de Crotalaria spectabilis e Crotalaria ochroleuca.

\section{Referências}

ARIZALETA, M. et al. Efecto del uso de metsulfuron-metil y glifosato sobre malezas associados a cafetales en Venezuela. Bioagro, v.20, n.2, p.79-88, 2008.

ASKEW, S.D. et al. Weed management in peanut (Arachis hypogea) with flumioxazin preemergence. Weed Technology, v.13, n.3, p.594-598, 1999.

BELANI, R.B. et al. Efeito de kixor em associação com glyphosate para controle de buva em dessecação pré-plantio da soja. In: CONGRESSO BRASILEIRO DA CIÊNCIA DAS PLANTAS DANINHAS, 27., 2010, Ribeirão Preto. Resumos... Ribeirão Preto: SBCPD, 2010. p.2367-2371.

BOGHOSSIAN, M.R. et al. Aspectos clínicopatológicos da intoxicação experimental pelas sementes de Crotalaria mucronata (Fabaceae) em bovinos. Pesquisa Veterinária Brasileira, v.27, n.4, p.149-156, 2007.

CARVALHO, F.T. et al. Manejo químico das plantas daninhas Euphorbia heterophylla e Bidens pilosa em sistema de plantio direto da cultura de soja. Planta Daninha, v.21, n.1, p.145-150, 2003.

CARVALHO, S.J.P.; LÓPEZ-OVEJERO, R.F. Resistência de plantas daninhas aos herbicidas inibidores da PROTOX. In: CHRISTOFFOLETI, P.J. (Ed.) Aspectos de Resistência de Plantas Daninhas a Herbicidas. Piracicaba: Associação Brasileira de Ação à Resistência de Plantas aos Herbicidas, 2008. cap. 5, p. 69-77,

CORREIA, N.M.; DURIGAN, J.C.; LEITE, G.J. Seletividade da soja transgênica tolerante ao glyphosate e eficácia de controle de Commelina benghalensis com herbicidas aplicados isolados e em misturas. Bragantia, v.67, n.3, p.563-568, 2008. 
EMBRAPA, Empresa Brasileira de Pesquisa Agropecuária. Centro Nacional de Pesquisa de Solos (Rio de Janeiro). Sistema Brasileiro de Classificação de solos. 2 ed. Rio de Janeiro: Embrapa, 2006. 306p.

ERASMO, E.A.L. et al. Potencial de espécies utilizadas como adubo verde no manejo integrado de plantas daninhas. Planta Daninha, v.22, n.3, p.337-342, 2004.

ESPANHOL, M. et al. Dessecação de Crotalaria juncea no outono. Revista Brasileira de Ciências Agrárias, v.6, n.1, p.90-97, 2011.

FORNAROLLI, D.A. et al. Eficácia do herbicida kixor no controle das espécies Ipomoea triloba e Bidens pilosa na região de Campo Mourão. In: CONGRESSO BRASILEIRO DA CIÊNCIA DAS PLANTAS DANINHAS, 27., 2010, Ribeirão Preto. Resumos... Ribeirão Preto: SBCPD, 2010. p.22-25.

HAYASHI, M.M.S. et al. Balanço de energia da Crotalaria juncea L. no período seco e no período úmido do ano, em condições de Cerrado. Revista Brasileira de Agrometeorologia, v.10, n.2, p.197-205, 2002.

INOMOTO, M.M. et al. Avaliação em casa de vegetação do uso de sorgo, milheto e crotalária no manejo de Meloidogyne javanica. Tropical Plant Pathology, v.33, n.2, p.125-129, 2008.

MONQUERO, P.A. et al. Glyphosate em mistura com herbicidas alternativos para o manejo de plantas daninhas. Planta Daninha, v.19, n.3, p.233-238, 2001.

NASCIMENTO, J.T. et al. Efeito de leguminosas nos atributos físicos e carbono orgânico de um Luvissolo. Revista Brasileira de Ciência do Solo, v.29, n.5, p.825-831, 2005.

NIEKAMP, J.W. et al. Broadleaf weed control with sulfentrazone and flumioxazin in no tillage soybean (Glycine max). Weed Technology, v.13, n.2, p.233-238, 1999.
OLIVEIRA NETO, A.M. et al. Manejo químico de adubos verdes para sucessão da cana-de-açúcar em sistema de cultivo mínimo.

Revista Brasileira de Herbicidas, v.10, n.2, p.86-94, 2011.

PROCÓPIO, S.O. et al. Efeitos de dessecantes no controle de plantas daninhas na cultura da soja. Planta Daninha, v.24, n.1, p.193-197, 2006.

RODRIGUES, B.N.; ALMEIDA, F.S. Guia de herbicidas. 6. ed. Londrina IAPAR, 2011, $697 p$.

SANTOS, V.S.; CAMPELO JR., J.J.H. Influência dos elementos meteorológicos na produção de adubos verdes, em diferentes épocas de semeadura. Revista Brasileira de Engenharia Agrícola e Ambiental, v.7, n.1, p.91-98, 2003.

SBCPD, Sociedade Brasileira da Ciência das Plantas Daninhas. Procedimentos para instalação, avaliação e análise de experimentos com herbicidas. Londrina: SBCPD, 1995. 42p.

SCHERDER, E.F. et al. Antagonism of cyhalofop grass activity by halosulfuron, triclopyr and propanil. Weed Technology, v.19, n.4, p.934-941, 2005. 